\title{
The Potential of Medical Consent Contracts Using Visuals
}

\author{
Nin Kirkham and Camilla B Andersen* \\ Department of Arts, University of Western Australia, Australia
}

*Corresponding author: Camilla B Andersen, Department of Arts, Business, Law and Education, University of Western Australia, Australia.

To Cite This Article: Camilla B Andersen, The Potential of Medical Consent Contracts Using Visuals. 2020 - 8(3). AJBSR.MS.ID.001261. DOI: 10.34297/AJBSR.2020.08.001261

Received: 眥 March 12, 2020; Published: 紫 March 19, 2020

\begin{abstract}
The ethical and legal requirement to gain informed consent from patients for medical procedures and for biomedical research faces challenges with respect to the transparency problem. The requirement to be transparent by using plain, non-technical language in their Patient Informed Consent Forms (PICFs) is clear but, in practice, hard to achieve. Problems of clear and transparent communication continue to provide a stumbling block to the ethical practice of medicine, law, and biomedical research. Research from the UWA Comic Contracting project indicates that visual/ graphic contracts are significantly better received by users in terms of their comprehension, engagement with the contract, and their perception of the other party. On this basis, we thus suggest that such developments might productively be applied to consent forms in medical and biomedical contexts in order to aid transparency and clarify communication of complicated concepts and language.
\end{abstract}

\section{Problems with Patient Informed Consent Forms}

In the context of the practices of medicine and biomedical research, 'informed consent' is required in order to respect the autonomy and dignity of patients and participants [1]. Hence, in order to fulfil the ethical maxim that we show respect for persons, informed consent practices are supposed to facilitate the complete transfer of information between two parties, e.g. a doctor and patient, or researcher and participant [2]. Tom Beauchamp explains that 'A person gives an informed consent...if and only if the person, with substantial understanding and in substantial absence of control by others, intentionally authorizes a health professional to do something' [3]. In biomedical contexts, this transfer of information allows a patient to become aware of any potential risks that they may face in consenting to a procedure and to consider whether and how consenting to the procedure may engender a conflict with their values or their preferences. Practitioners and researchers must ask themselves: 'would a reasonable person, in the position of the patient, be likely to attach significance to the risk?' and 'Is the doctor aware, or should the doctor be reasonably aware, that this particular patient would be likely to attach significance to that risk?' [4]. Ideally, practitioners and researchers should make themselves aware of any power imbalances that may arise between them and their patients and participant [5], because a patient's decision-making ability can be affected by who it is that is informing them [6].

Further, and of central importance in the context of this paper, practitioners are encouraged to check if their patients have truly understood what they have been told. One way this can be done is to ask patients to explain, in their own words, what a certain procedure involves. But an epistemic problem arises because some details of medical and biomedical research practices can remain partially or completely opaque even to well informed patients and participantswe might call this potential for ineffective or incomplete transfer of crucial information the transparency (or explanation) problem. The problem is especially pressing because receiving a clear and transparent explanation is a necessary condition for developing the ability to make an informed decision about any procedure to which one might be asked to give consent.

Even when practitioners and researchers make concerted efforts to be transparent, problems concerning clear communication can persist. Consent forms may be long; and even when they don't include hyper technical jargon, they still may be written 
using a technical language that is hard to interpret, making them inaccessible to many people who may not be inclined to, or not in the practice of, carefully reading long, complex documents. It is at this juncture of the informed consent process that we believe the insights from developments of comic book contracts may be usefully applied.

\section{Potential of Visual Imagery to Improve PICFs}

It is of crucial importance, thus, that an 'information transfer' occurs, between patients/research participants and doctors/ researchers. An alternative approach to the standard consent forms could make use of some of the latest research focused on the usability of pictorial legal contracts. There is some movement in the UK on the use of YouTube videos and illustrated forms for infographics, but the law has been slow to embrace these developments, seeing information illustrated but legal forms typically NOT [7]. Thus, sadly, most medical consent forms continue to be in the medical or legal jargon which tends to alienate users.

The use of visuals in legally binding contracts has been emerging in several disciplines and in distinct parts of the world. Lawyer Robert de Roy has created employment contracts which are predominantly in cartoon format, aimed at illiterate farm workers, and as such has been recognized by the Hague Institute for the Innovation in Law (HiiL) as an important initiative in access to justice [8]. Simultaneously, here at UWA, the Comic Contract project has developed several iterations of different contracts, all highly visual to different degrees and styles [9]. Over a longitudinal impact study, these comic contracts have proven to be effective in advancing not only comprehension of contacts, but also engagement with and perception of the contractual relationship.

With regards to comprehension, the project tested people under the old text-based contract versus new employees under the visual contract. The weighted percent increase in comprehension feedback was $9.17 \%$ across the different groups, indicating a significant increase. Based on the earlier research of Stefania Passera, we largely expected this. However, the data on engagement and perception was even more gratifying. Across the different groupings of employees, there was a significant increase in the engagement with the contract, even higher than seen for comprehension, with a total of $13.24 \%$ weighted point difference measured. This means that the people who engaged with the visual and smart-linked comic contract were significantly more engaged in the process, and captivated by the information they were immersing themselves in.

Moreover, a staggering $15.17 \%$ weighted point difference indicated a significant preference for the comic contract in terms of perceiving the company favorably for producing one and making the effort to design a more friendly format. The team was initially surprised by this, as we had assumed the greatest increases were to be found in comprehension, based on the established scientific data in this field. However, the project has indicated strongly that comprehension is only a small part of the advantage's comic contracting has to offer.

Given an essential part of informed consent involves a transfer of information, and an engagement in a process which needs to be perceived well, comic contracts may help due to their ability to simplify complex information. There is, after all, evidence to suggest that by simplifying medical consent decrees, a greater comprehension and retention of information ensues. Further, the visual aspect of comic contracts may also help with basic comprehension, since there is evidence to suggest that visual explanations can improve learning. We do not claim that pictorial PICFs are likely to be a 'magic bullet' but visuals can become an important part of transparency and engagement. It is for this reason that the comic contracting project is now striking into this new territory and hoping to report on the impact of visual legal consent forms within the near future.

\section{References}

1. Beauchamp, Tom L (2011) Informed Consent: Its History, Meaning, and Present Challenges. Cambridge Quarterly of Healthcare Ethics 20(4): 515-23.

2. Bobek E, Tversky B (2016) Creating visual explanations improves learning. Cognitive Research: Principles and Implications 1(1): 27.

3. Dresden GM, Levitt MA (2001) Modifying a standard industry clinical trial consent form improves patient information retention as part of the informed consent process. Acad Emerg Med 8: 246-252.

4. Kadam RA (2017) Informed consent process: A step further towards making it meaningful. Perspect Clin Res 8(3): 107-112.

5. Manson N, Onora O Neill (2007) Rethinking Informed Consent in Bioethics. Cambridge University Press, United Kingdom.

6. Nimmon L, Stenfors Hayes T (2016) The Handling of power in the physician patient encounter perceptions from experienced physicians. Bmc Medical Education 16(1): 114.

7. Passera S (2017) Beyond the wall of contract text, Aalto Dissertation Press, Espoo, Finland pp.221.

8. RAGCP (2019) Informed Consent: Information Sheet, RAGCP ORG, [Australia.

9. RANZCOG (2018) RANZCOG Medical Schools Curriculum in Obstetrics \& Gynecology (AMC Alignment). The Royal Australian and New Zealand College of Obstetricians and Gynaecologists, Albert Street, East Melbourne, Australia pp. 254-260. 